\title{
Ulam stabilities for partial Hadamard fractional integral equations
}

Received: 13 September 2015 / Accepted: 13 December 2015 / Published online: 12 January 2016

(C) The Author(s) 2016. This article is published with open access at Springerlink.com

\begin{abstract}
This paper deals with some existence and Ulam stability results for a class of partial integral equations via Hadamard's fractional integral, by applying Schauder's fixed-point theorem.

Mathematics Subject Classification 34A08 · 34K05

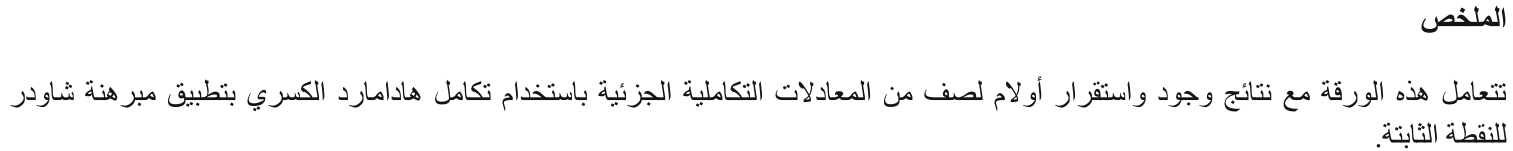

\section{Introduction}

The fractional calculus represents a powerful tool in applied mathematics to study many problems from different fields of science and engineering, with many breakthroughs in mathematical physics, finance, hydrology, biophysics, thermodynamics, control theory, statistical mechanics, astrophysics, cosmology and bioengineering $[13,27]$. There has been a significant development in ordinary and partial fractional differential and integral equations in recent years; see the monographs of Abbas et al. [3,4], Kilbas et al. [19], Miller and Ross [20], the papers of Abbas and Benchohra [1,2], Abbas et al. [5], Benchohra et al. [6], Vityuk [29], Vityuk and Golushkov [30] and the references therein.

In [8], Butzer et al. investigated properties of the Hadamard fractional integral and the derivative. In [9], they obtained the Mellin transforms of the Hadamard fractional integral and differential operators and in [22], Pooseh et al. obtained expansion formulas of the Hadamard operators in terms of integer-order derivatives.

\section{S. Abbas}

Laboratory of Mathematics, University of Saïda, P.O. Box 138, 20000 Saida, Algeria

E-mail: abbasmsaid@yahoo.fr

\section{Benchohra $(\varangle)$}

Laboratory of Mathematics, University of Sidi Bel-Abbès, P.O. Box 89, Sidi Bel-Abbès 22000, Algeria

E-mail: benchohra@yahoo.com; benchohra@univ-sba.dz

M. Benchohra · W. Albarakati

Department of Mathematics, Faculty of Science, King Abdulaziz University, P.O. Box 80203, Jeddah 21589, Saudi Arabia

E-mail: wbarakati@kau.edu.sa

J. J. Trujillo

Departamento de Análisis Matemático, Universidad de La Laguna, 38271 La Laguna, Tenerife, Spain

E-mail: jtrujill@ullmat.es 
Many other interesting properties of those operators and others are summarized in [26] and the references therein.

The stability of functional equations was originally raised by Ulam in 1940 in a talk given at Wisconsin University. The problem posed by Ulam was the following: Under what conditions does there exist an additive mapping near an approximately additive mapping? (for more details see [28]). The first answer to Ulam's question was given by Hyers in 1941 in the case of Banach spaces in [14]. Thereafter, this type of stability is called the Ulam-Hyers stability. In 1978, Rassias [23] provided a remarkable generalization of the Ulam-Hyers stability of mappings by considering variables. The concept of stability for a functional equation arises when we replace the functional equation by an inequality which acts as a perturbation of the equation. Thus, the stability question of functional equations is how do the solutions of the inequality differ from those of the given functional equation? Considerable attention has been given to the study of the Ulam-Hyers and Ulam-HyersRassias stability of all kinds of functional equations; one can see the monographs [15,16]. Bota-Boriceanu and Petrusel [7], Petru et al. [21], and Rus [24,25] discussed the Ulam-Hyers stability for operatorial equations and inclusions. Castro and Ramos [10] and Jung [18] considered the Hyers-Ulam-Rassias stability for a class of Volterra integral equations. More details from historical point of view, and recent developments of such stabilities are reported in [17,24].

This paper deals with the existence for the Ulam stability of solutions to the following Hadamard partial fractional integral equation of the form

$$
\begin{gathered}
u(x, y)=\mu(x, y) \\
+\frac{1}{\Gamma\left(r_{1}\right) \Gamma\left(r_{2}\right)} \int_{1}^{x} \int_{1}^{y}\left(\log \frac{x}{s}\right)^{r_{1}-1}\left(\log \frac{y}{t}\right)^{r_{2}-1} \frac{f(s, t, u(s, t))}{s t} \mathrm{~d} t \mathrm{~d} s ; \quad \text { if }(x, y) \in J,
\end{gathered}
$$

where $J:=[1, a] \times[1, b], a, b>1, r_{1}, r_{2}>0, \mu: J \rightarrow \mathbb{R}, f: J \times \mathbb{R} \rightarrow \mathbb{R}$ are given continuous functions.

We present two results for the integral equation (1). The first one is based on Banach's contraction principle and the second one on the nonlinear alternative of Leray-Schauder type.

The present paper initiates the Ulam stability for integral equations involving the Hadamard fractional integral.

\section{Preliminaries}

In this section, we introduce notations, definitions, and preliminary facts which are used throughout this paper. Denote by $C:=C(J, \mathbb{R})$ the Banach space of continuous functions $u: J \rightarrow \mathbb{R}$ with the norm

$$
\|u\|_{C}=\sup _{(x, y) \in J}|u(x, y)| .
$$

$L^{1}(J, \mathbb{R})$ the Banach space of functions $u: J \rightarrow \mathbb{R}$ that are Lebesgue integrable with norm

$$
\|u\|_{L^{1}}=\int_{1}^{a} \int_{1}^{b}|u(x, y)| d y d x .
$$

Definition 2.1 $[12,19]$ The Hadamard fractional integral of order $q>0$ for a function $g \in L^{1}([1, a]$, $\mathbb{R})$, is defined as

$$
\left({ }^{H} I_{1}^{r} g\right)(x)=\frac{1}{\Gamma(q)} \int_{1}^{x}\left(\log \frac{x}{s}\right)^{q-1} \frac{g(s)}{s} \mathrm{~d} s,
$$

where $\Gamma(\cdot)$ is the Euler gamma function.

Definition 2.2 Let $r_{1}, r_{2} \geq 0, \sigma=(1,1)$ and $r=\left(r_{1}, r_{2}\right)$. For $w \in L^{1}(J, \mathbb{R})$, define the Hadamard partial fractional integral of order $r$ by the expression

$$
\left({ }^{H} I_{\sigma}^{r} w\right)(x, y)=\frac{1}{\Gamma\left(r_{1}\right) \Gamma\left(r_{2}\right)} \int_{1}^{x} \int_{1}^{y}\left(\log \frac{x}{s}\right)^{r_{1}-1}\left(\log \frac{y}{t}\right)^{r_{2}-1} \frac{w(s, t)}{s t} \mathrm{~d} t \mathrm{~d} s .
$$

Now, we consider the Ulam stability for the integral equation (1). Consider the operator $N: C \rightarrow C$ defined by: 


$$
(N u)(x, y)=\mu(x, y)+\frac{1}{\Gamma\left(r_{1}\right) \Gamma\left(r_{2}\right)} \int_{1}^{x} \int_{1}^{y}\left(\log \frac{x}{s}\right)^{r_{1}-1}\left(\log \frac{y}{t}\right)^{r_{2}-1} \frac{f(s, t, u(s, t))}{s t} \mathrm{~d} t \mathrm{~d} s .
$$

Clearly, the fixed points of the operator $N$ are solution of the integral equation (1). Let $\epsilon>0$ and $\Phi: J \rightarrow$ $[0, \infty)$ be a continuous function. We consider the following inequalities

$$
\begin{gathered}
|u(x, y)-(N u)(x, y)| \leq \epsilon ;(x, y) \in J . \\
|u(x, y)-(N u)(x, y)| \leq \Phi(x, y) ; \quad(x, y) \in J \\
|u(x, y)-(N u)(x, y)| \leq \epsilon \Phi(x, y) ;(x, y) \in J .
\end{gathered}
$$

Definition 2.3 [3,24] Equation (1) is Ulam-Hyers stable if there exists a real number $c_{N}>0$ such that for each $\epsilon>0$ and for each solution $u \in C$ of the inequality (3) there exists a solution $v \in C$ of Eq. (1) with

$$
|u(x, y)-v(x, y)| \leq \epsilon c_{N} ; \quad(x, y) \in J .
$$

Definition 2.4 $[3,24]$ Equation (1) is generalized Ulam-Hyers stable if there exists $c_{N}: C([0, \infty),[0, \infty))$ with $c_{N}(0)=0$ such that for each $\epsilon>0$ and for each solution $u \in \mathcal{C}$ of the inequality (3) there exists a solution $v \in C$ of Eq. (1) with

$$
|u(x, y)-v(x, y)| \leq c_{N}(\epsilon) ; \quad(x, y) \in J .
$$

Definition 2.5 [3,24] Equation (1) is Ulam-Hyers-Rassias stable with respect to $\Phi$ if there exists a real number $c_{N, \Phi}>0$ such that for each $\epsilon>0$ and for each solution $u \in C$ of the inequality (5) there exists a solution $v \in C$ of Eq. (1) with

$$
|u(x, y)-v(x, y)| \leq \epsilon c_{N, \Phi} \Phi(x, y) ; \quad(x, y) \in J .
$$

Definition 2.6 [3,24] Equation (1) is generalized Ulam-Hyers-Rassias stable with respect to $\Phi$ if there exists a real number $c_{N, \Phi}>0$ such that for each solution $u \in C$ of the inequality (4) there exists a solution $v \in \mathcal{C}$ of Eq. (1) with $|u(x, y)-v(x, y)| \leq c_{N, \Phi} \Phi(x, y) ;(x, y) \in J$.

Remark 2.7 It is clear that (i) Definition $2.3 \Rightarrow$ Definition 2.4, (ii) Definition $2.5 \Rightarrow$ Definition 2.6, (iii) Definition 2.5 for $\Phi(.,)=.1 \Rightarrow$ Definition 2.3 .

One can have similar remarks for the inequalities (3) and (5).

\section{Existence and Ulam stabilities results}

In this section, we discuss the existence of solutions and present conditions for the Ulam stability for the Hadamard integral equation (1).

The following hypotheses will be used in the sequel.

$\left(H_{1}\right)$ There exist functions $p_{1}, p_{2} \in C\left(J, \mathbb{R}_{+}\right)$such that for any $u \in \mathbb{R}$ and $(x, y) \in J$,

$$
|f(x, y, u)| \leq p_{1}(x, y)+\frac{p_{2}(x, y)}{1+|u(x, y)|}|u(x, y)|,
$$

with

$$
p_{i}^{*}=\sup _{(x, y) \in J} p_{i}(x, y) ; i=1,2,
$$

$\left(H_{2}\right)$ There exists $\lambda_{\Phi}>0$ such that for each $(x, y) \in J$, we have

$$
\left({ }^{H} I_{\sigma}^{r} \Phi\right)(x, y) \leq \lambda_{\Phi} \Phi(x, y) .
$$

Theorem 3.1 Assume that the hypothesis $\left(H_{1}\right)$ holds. If

$$
\frac{(\log a)^{r_{1}}(\log b)^{r_{2}}}{\Gamma\left(1+r_{1}\right) \Gamma\left(1+r_{2}\right)} p_{2}^{*}<1,
$$

then the integral equation (1) has a solution defined on J. 
Proof Let $\rho>0$ be a constant such that

$$
\rho>\frac{\|\mu\|_{\infty}+\frac{(\log a)^{r_{1}}(\log b)^{r_{2}}}{\Gamma\left(1+r_{1}\right) \Gamma\left(1+r_{2}\right)} p_{1}^{*}}{1-\frac{(\log a)^{r_{1}}(\log b)^{r_{2}}}{\Gamma\left(1+r_{1}\right) \Gamma\left(1+r_{2}\right)} p_{2}^{*}} .
$$

We shall use Schauder's theorem [11], to prove that the operator $N$ defined in (2) has a fixed point. The proof will be given in four steps.

Step 1: $N$ transforms the ball $B_{\rho}:=\left\{u \in \mathcal{C}:\|u\|_{C} \leq \rho\right\}$ into itself.

For any $u \in B_{\rho}$ and each $(x, y) \in J$, we have

$$
\begin{aligned}
|(N u)(x, y)| \leq & |\mu(x, y)|+\frac{1}{\Gamma\left(r_{1}\right) \Gamma\left(r_{2}\right)} \int_{1}^{x} \int_{1}^{y}\left|\log \frac{x}{s}\right|^{r_{1}-1}\left|\log \frac{y}{t}\right|^{r_{2}-1} \\
& \times \frac{p_{1}(s, t)+p_{2}(s, t)\|u\|_{C}}{s t} \mathrm{~d} t \mathrm{~d} s \\
\leq & \|\mu\|_{\infty}+\frac{(\log a)^{r_{1}}(\log b)^{r_{2}}}{\Gamma\left(1+r_{1}\right) \Gamma\left(1+r_{2}\right)}\left(p_{1}^{*}+p_{2}^{*} \rho\right) .
\end{aligned}
$$

Thus, by (6) and the definition of $\rho$ we get $\|(N u)\|_{C} \leq \rho$. This implies that $N$ transforms the ball $B_{\rho}$ into itself.

Step 2: $N: B_{\rho} \rightarrow B_{\rho}$ is continuous.

Let $\left\{u_{n}\right\}_{n \in \mathbb{N}}$ be a sequence such that $u_{n} \rightarrow u$ in $B_{\rho}$. Then

$$
\begin{aligned}
\left|\left(N u_{n}\right)(x, y)-(N u)(x, y)\right| \leq & \frac{1}{\Gamma\left(r_{1}\right) \Gamma\left(r_{2}\right)} \int_{1}^{x} \int_{1}^{y}\left|\log \frac{x}{s}\right|^{r_{1}-1}\left|\log \frac{y}{t}\right|^{r_{2}-1} \\
& \times \frac{\left|f\left(s, t, u_{n}(s, t)\right)-f(s, t, u(s, t))\right|}{\mathrm{d} t \mathrm{~d} s} \\
\leq & \frac{1}{\Gamma\left(r_{1}\right) \Gamma\left(r_{2}\right)} \int_{1}^{x} \int_{1}^{y}\left|\log \frac{x}{s}\right|^{r_{1}-1}\left|\log \frac{y}{t}\right|^{r_{2}-1} \\
& \times \frac{\sup _{(s, t) \in J}\left|f\left(s, t, u_{n}(s, t)\right)-f(s, t, u(s, t))\right|}{\leq} \mathrm{d} t \mathrm{~d} s \\
\leq & \frac{(\log a)^{r_{1}}(\log b)^{r_{2}}}{\Gamma\left(1+r_{1}\right) \Gamma\left(1+r_{2}\right)}\left\|f\left(\cdot, \cdot, u_{n}(\cdot, \cdot)\right)-f(\cdot, \cdot, u(\cdot, \cdot))\right\|_{C} .
\end{aligned}
$$

From Lebesgue's dominated convergence theorem and the continuity of the function $f$, we get

$$
\left|\left(N u_{n}\right)(x, y)-(N u)(x, y)\right| \rightarrow 0 \quad \text { as } \quad n \rightarrow \infty .
$$

Step 3: $N\left(B_{\rho}\right)$ is bounded.

This is clear since $N\left(B_{\rho}\right) \subset B_{\rho}$ and $B_{\rho}$ is bounded.

Step 4: $N\left(B_{\rho}\right)$ is equicontinuous.

Let $\left(x_{1}, y_{1}\right),\left(x_{2}, y_{2}\right) \in J, x_{1}<x_{2}, y_{1}<y_{2}$. Then

$$
\begin{aligned}
& \left|(N u)\left(x_{2}, y_{2}\right)-(N u)\left(x_{1}, y_{1}\right)\right| \leq\left|\mu\left(x_{1}, y_{1}\right)-\mu\left(x_{2}, y_{2}\right)\right| \\
& +\frac{1}{\Gamma\left(r_{1}\right) \Gamma\left(r_{2}\right)} \int_{1}^{x_{1}} \int_{1}^{y_{1}}\left[\left|\log \frac{x_{2}}{s}\right|^{r_{1}-1}\left|\log \frac{y_{2}}{t}\right|^{r_{2}-1}-\left|\log \frac{x_{1}}{s}\right|^{r_{1}-1}\left|\log \frac{y_{1}}{t}\right|^{r_{2}-1}\right] \\
& \quad \times \frac{|f(s, t, u(s, t))|}{s t} \mathrm{~d} t \mathrm{~d} s \\
& +\frac{1}{\Gamma\left(r_{1}\right) \Gamma\left(r_{2}\right)} \int_{x_{1}}^{x_{2}} \int_{y_{1}}^{y_{2}}\left|\log \frac{x_{2}}{s}\right|^{r_{1}-1}\left|\log \frac{y_{2}}{t}\right|^{r_{2}-1} \frac{|f(s, t, u(s, t))|}{s t} \mathrm{~d} t \mathrm{~d} s \\
& +\frac{1}{\Gamma\left(r_{1}\right) \Gamma\left(r_{2}\right)} \int_{1}^{x_{1}} \int_{y_{1}}^{y_{2}}\left|\log \frac{x_{2}}{s}\right|^{r_{1}-1}\left|\log \frac{y_{2}}{t}\right|^{r_{2}-1} \frac{|f(s, t, u(s, t))|}{s t} \mathrm{~d} t \mathrm{~d} s \\
& +\frac{1}{\Gamma\left(r_{1}\right) \Gamma\left(r_{2}\right)} \int_{x_{1}}^{x_{2}} \int_{1}^{y_{1}}\left|\log \frac{x_{2}}{s}\right|^{r_{1}-1}\left|\log \frac{y_{2}}{t}\right|^{r_{2}-1} \frac{|f(s, t, u(s, t))|}{s t} \mathrm{~d} t \mathrm{~d} s .
\end{aligned}
$$


Thus

$$
\begin{aligned}
& \left|(N u)\left(x_{2}, y_{2}\right)-(N u)\left(x_{1}, y_{1}\right)\right| \leq\left|\mu\left(x_{1}, y_{1}\right)-\mu\left(x_{2}, y_{2}\right)\right| \\
& +\frac{1}{\Gamma\left(r_{1}\right) \Gamma\left(r_{2}\right)} \int_{1}^{x_{1}} \int_{1}^{y_{1}}\left[\left|\log \frac{x_{2}}{s}\right|^{r_{1}-1}\left|\log \frac{y_{2}}{t}\right|^{r_{2}-1}-\left|\log \frac{x_{1}}{s}\right|^{r_{1}-1}\left|\log \frac{y_{1}}{t}\right|^{r_{2}-1}\right] \\
& \times \frac{p_{1}^{*}+p_{2}^{*} \rho}{s t} \mathrm{~d} t \mathrm{~d} s \\
& +\frac{1}{\Gamma\left(r_{1}\right) \Gamma\left(r_{2}\right)} \int_{x_{1}}^{x_{2}} \int_{y_{1}}^{y_{2}}\left|\log \frac{x_{2}}{s}\right|^{r_{1}-1}\left|\log \frac{y_{2}}{t}\right|^{r_{2}-1} \frac{p_{1}^{*}+p_{2}^{*} \rho}{s t} \mathrm{~d} t \mathrm{~d} s \\
& +\frac{1}{\Gamma\left(r_{1}\right) \Gamma\left(r_{2}\right)} \int_{1}^{x_{1}} \int_{y_{1}}^{y_{2}}\left|\log \frac{x_{2}}{s}\right|^{r_{1}-1}\left|\log \frac{y_{2}}{t}\right|^{r_{2}-1} \frac{p_{1}^{*}+p_{2}^{*} \rho}{s t} \mathrm{~d} t \mathrm{~d} s \\
& +\frac{1}{\Gamma\left(r_{1}\right) \Gamma\left(r_{2}\right)} \int_{x_{1}}^{x_{2}} \int_{1}^{y_{1}}\left|\log \frac{x_{2}}{s}\right|^{r_{1}-1}\left|\log \frac{y_{2}}{t}\right|^{r_{2}-1} \frac{p_{1}^{*}+p_{2}^{*} \rho}{s t} \mathrm{~d} t \mathrm{~d} s \\
& \leq \frac{p_{1}^{*}+p_{2}^{*} \rho}{\Gamma\left(1+r_{1}\right) \Gamma\left(1+r_{2}\right)} \\
& \times\left[2\left(\log y_{2}\right)^{r_{2}}\left(\log x_{2}-\log x_{1}\right)^{r_{1}}+2\left(\log x_{2}\right)^{r_{1}}\left(\log y_{2}-\log y_{1}\right)^{r_{2}}\right. \\
& \left.+\left(\log x_{1}\right)^{r_{1}}\left(\log y_{1}\right)^{r_{2}}-\left(\log x_{2}\right)^{r_{1}}\left(\log y_{2}\right)^{r_{2}}-2\left(\log x_{2}-\log x_{1}\right)^{r_{1}}\left(\log y_{2}-\log y_{1}\right)^{r_{2}}\right] \text {. }
\end{aligned}
$$

As $x_{1} \rightarrow x_{2}$ and $y_{1} \rightarrow y_{2}$, the right-hand side of the above inequality tends to zero.

As a consequence of steps 1-4 together with the Arzelá-Ascoli theorem, we can conclude that $N$ is continuous and compact. From an application of Schauder's theorem [11], we deduce that $N$ has a fixed point $u$ which is a solution of the integral equation (1).

Now, we are concerned with the stability of solutions for the integral equation (1).

Theorem 3.2 Assume that $\left(H_{1}\right),\left(H_{2}\right)$ and the condition (6) hold. Furthermore, suppose that there exist $q_{i} \in$ $C\left(J, \mathbb{R}_{+}\right) ; i=1,2$ such that for each $(x, y) \in J$ we have

$$
p_{i}(x, y) \leq q_{i}(x, y) \Phi(x, y) .
$$

Then, the integral equation (1) is generalized Ulam-Hyers-Rassias stable.

Proof Let $u$ be a solution of the inequality (4). By Theorem 3.1, there exists $v$ which is a solution of the integral equation (1). Hence

$$
v(x, y)=\mu(x, y)+\int_{1}^{x} \int_{1}^{y}\left(\log \frac{x}{s}\right)^{r_{1}-1}\left(\log \frac{y}{t}\right)^{r_{2}-1} \frac{f(s, t, v(s, t))}{s t \Gamma\left(r_{1}\right) \Gamma\left(r_{2}\right)} \mathrm{d} t \mathrm{~d} s .
$$

By the inequality (4) for each $(x, y) \in J$, we have

$$
\left|u(x, y)-\mu(x, y)-\int_{1}^{x} \int_{1}^{y}\left(\log \frac{x}{s}\right)^{r_{1}-1}\left(\log \frac{y}{t}\right)^{r_{2}-1} \frac{f(s, t, u(s, t))}{s t \Gamma\left(r_{1}\right) \Gamma\left(r_{2}\right)} \mathrm{d} t \mathrm{~d} s\right| \leq \Phi(x, y) .
$$

Set

$$
q_{i}^{*}=\sup _{(x, y) \in J} q_{i}(x, y) ; \quad i=1,2
$$


For each $(x, y) \in J$, we have

$$
\begin{aligned}
&|u(x, y)-v(x, y)| \\
& \leq\left|u(x, y)-\mu(x, y)-\int_{1}^{x} \int_{1}^{y}\left(\log \frac{x}{s}\right)^{r_{1}-1}\left(\log \frac{y}{t}\right)^{r_{2}-1} \frac{f(s, t, u(s, t))}{s t \Gamma\left(r_{1}\right) \Gamma\left(r_{2}\right)} \mathrm{d} t \mathrm{~d} s\right| \\
& \quad+\int_{1}^{x} \int_{1}^{y}\left|\log \frac{x}{s}\right|^{r_{1}-1}\left|\log \frac{y}{t}\right|^{r_{2}-1} \frac{|f(s, t, u(s, t))-f(s, t, v(s, t))|}{s t \Gamma\left(r_{1}\right) \Gamma\left(r_{2}\right)} \mathrm{d} t \mathrm{~d} s \\
& \leq \Phi(x, y)+\frac{1}{\Gamma\left(r_{1}\right) \Gamma\left(r_{2}\right)} \int_{1}^{x} \int_{1}^{y}\left|\log \frac{x}{s}\right|^{r_{1}-1}\left|\log \frac{y}{t}\right|^{r_{2}-1} \\
& \times\left(2 q_{1}^{*}+\frac{q_{2}^{*}|u(s, t)|}{1+|u|}+\frac{q_{2}^{*}|v(s, t)|}{1+|v|}\right) \frac{\Phi(s, t)}{s t} \mathrm{~d} t \mathrm{~d} s \\
& \leq \Phi(x, y)+2\left(q_{1}^{*}+q_{2}^{*}\right)\left({ }^{H} I_{\sigma}^{r} \Phi\right)(x, y) \\
& \leq {\left[1+2\left(q_{1}^{*}+q_{2}^{*}\right) \lambda_{\phi}\right] \Phi(x, y) } \\
&:= c_{N, \Phi} \Phi(x, y) .
\end{aligned}
$$

Hence, the integral equation (1) is generalized Ulam-Hyers-Rassias stable.

\section{An example}

As an application of our results, we consider the following partial Hadamard integral equation

$$
\begin{aligned}
u(x, y)= & \mu(x, y) \\
& +\int_{1}^{x} \int_{1}^{y}\left(\log \frac{x}{s}\right)^{r_{1}-1}\left(\log \frac{y}{t}\right)^{r_{2}-1} \frac{f(s, t, u(s, t))}{s t \Gamma\left(r_{1}\right) \Gamma\left(r_{2}\right)} \mathrm{d} t \mathrm{~d} s ; \quad(x, y) \in[1, e] \times[1, e],
\end{aligned}
$$

where

$$
r_{1}, r_{2}>0, \quad \mu(x, y)=x+y^{2} ; \quad(x, y) \in[1, e] \times[1, e],
$$

and

$$
f(x, y, u(x, y))=c x y^{2}\left(e^{-4}+\frac{u(x, y)}{e^{x+y+5}}\right) ; \quad(x, y) \in[1, e] \times[1, e]
$$

with

$$
c:=\frac{e^{4}}{2} \Gamma\left(1+r_{1}\right) \Gamma\left(1+r_{2}\right) .
$$

For each $u \in \mathbb{R}$ and $(x, y) \in[1, e] \times[1, e]$ we have

$$
|f(x, y, u(x, y))| \leq c e^{-4}(1+|u|) .
$$

Hence, condition (6) is satisfied with $p_{1}^{*}=p_{2}^{*}=c e^{-4}$. We shall show that condition (6) holds with $a=b=e$. Indeed,

$$
\frac{(\log a)^{r_{1}}(\log b)^{r_{2}} p_{2}^{*}}{\Gamma\left(1+r_{1}\right) \Gamma\left(1+r_{2}\right)}=\frac{c}{e^{4} \Gamma\left(1+r_{1}\right) \Gamma\left(1+r_{2}\right)}=\frac{1}{2}<1 .
$$

Consequently, Theorem 3.1 implies that the Hadamard integral equation (7) has a solution defined on $[1, e] \times$ $[1, e]$.

Also, the hypothesis $\left(\mathrm{H}_{2}\right)$ is satisfied with

$$
\Phi(x, y)=e^{3}, \quad \text { and } \quad \lambda_{\Phi}=\frac{1}{\Gamma\left(1+r_{1}\right) \Gamma\left(1+r_{2}\right)} .
$$


Indeed, for each $(x, y) \in[1, e] \times[1, e]$ we get

$$
\begin{aligned}
\left({ }^{H} I_{\sigma}^{r} \Phi\right)(x, y) & \leq \frac{e^{3}}{\Gamma\left(1+r_{1}\right) \Gamma\left(1+r_{2}\right)} \\
& =\lambda_{\Phi} \Phi(x, y) .
\end{aligned}
$$

Consequently, Theorem 3.2 implies that the equation (7) is generalized Ulam-Hyers-Rassias stable.

Acknowledgments This work was partially funded by FEDER funds and project MTM2013-41704-P from the government of Spain.

Open Access This article is distributed under the terms of the Creative Commons Attribution 4.0 International License (http:// creativecommons.org/licenses/by/4.0/), which permits unrestricted use, distribution, and reproduction in any medium, provided you give appropriate credit to the original author(s) and the source, provide a link to the Creative Commons license, and indicate if changes were made.

\section{References}

1. Abbas, S.; Benchohra, M.: Partial hyperbolic differential equations with finite delay involving the Caputo fractional derivative. Commun. Math. Anal. 7, 62-72 (2009)

2. Abbas, S.; Benchohra, M.: Fractional order integral equations of two independent variables. Appl. Math. Comput. 227, 755-761 (2014)

3. Abbas, S.; Benchohra, M.; N'Guérékata, G.M.: Topics in Fractional Differential Equations. Springer, New York (2012)

4. Abbas, S.; Benchohra, M.; N'Guérékata, G.M.: Advanced Fractional Differential and Integral Equations. Nova Science Publishers, New York (2015)

5. Abbas, S.; Benchohra, M.; Vityuk, A.N.: On fractional order derivatives and Darboux problem for implicit differential equations. Fract. Calc. Appl. Anal. 15, 168-182 (2012)

6. Benchohra, M.; Henderson, J.; Ntouyas, S.K.; Ouahab, A.: Existence results for functional differential equations of fractional order. J. Math. Anal. Appl. 338, 1340-1350 (2008)

7. Bota-Boriceanu, M.F.; Petrusel, A.: Ulam-Hyers stability for operatorial equations and inclusions. Analele Univ. I. Cuza Iasi. 57, 65-74 (2011)

8. Butzer, P.L.; Kilbas, A.A.; Trujillo, J.J.: Fractional calculus in the Mellin setting and Hadamard-type fractional integrals. J. Math. Anal. Appl. 269, 1-27 (2002)

9. Butzer, P.L.; Kilbas, A.A.; Trujillo, J.J.: Mellin transform analysis and integration by parts for Hadamard-type fractional integrals. J. Math. Anal. Appl. 270, 1-15 (2002)

10. Castro, L.P.; Ramos, A.: Hyers-Ulam-Rassias stability for a class of Volterra integral equations. Banach J. Math. Anal. 3, 36-43 (2009)

11. Granas, A.; Dugundji, J.: Fixed Point Theory. Springer-Verlag, New York (2003)

12. Hadamard, J.: Essai sur l'étude des fonctions données par leur développment de Taylor. J. Pure Appl. Math. 4(8), 101-186 (1892)

13. Hilfer, R.: Applications of Fractional Calculus in Physics. World Scientific, Singapore (2000)

14. Hyers, D.H.: On the stability of the linear functional equation. Proc. Natl. Acad. Sci. 27, 222-224 (1941)

15. Hyers, D.H.; Isac, G.; Rassias, Th.M.: Stability of Functional Equations in Several Variables. Birkhuser (1998)

16. Jung, S.-M.: Hyers-Ulam-Rassias Stability of Functional Equations in Mathematical Analysis. Hadronic Press, Palm Harbor (2001)

17. Jung, S.-M.: Hyers-Ulam-Rassias Stability of Functional Equations in Nonlinear Analysis. Springer, New York (2011)

18. Jung, S.-M.: A fixed point approach to the stability of a Volterra integral equation. Fixed Point Theory Appl. 2007 (2007), Article ID 57064, 9 pages

19. Kilbas, A.A.; Srivastava, H.M.; Trujillo, J.J.: Theory and Applications of Fractional Differential Equations. Elsevier Science B.V.; Amsterdam (2006)

20. Miller, K.S.; Ross, B.: An Introduction to the Fractional Calculus and Differential Equations. John Wiley, New York (1993)

21. Petru, T.P.; Petrusel, A.; Yao, J.-C.: Ulam-Hyers stability for operatorial equations and inclusions via nonself operators. Taiwan. J. Math. 15, 2169-2193 (2011)

22. Pooseh, S.; Almeida, R.; Torres, D.: Expansion formulas in terms of integer-order derivatives for the Hadamard fractional integral and derivative. Numer. Funct. Anal. Optim. 33(3), 301-319 (2012)

23. Rassias, Th.M.; On the stability of linear mappings in Banach spaces. Proc. Am. Math. Soc. 72, 297-300 (1978)

24. Rus, I.A.: Ulam stability of ordinary differential equations. Studia Univ. Babes-Bolyai Math. LIV(4), 125-133 (2009)

25. Rus, I.A.: Remarks on Ulam stability of the operatorial equations. Fixed Point Theory 10, 305-320 (2009)

26. Samko, S.G.; Kilbas, A.A.; Marichev, O.I.: Fractional Integrals and Derivatives. Theory and Applications. Gordon and Breach, Yverdon (1993)

27. Tarasov, V.E.: Fractional Dynamics: Application of Fractional Calculus to Dynamics of Particles, Fields and Media. Springer, Heidelberg; Higher Education Press, Beijing (2010)

28. Ulam, S.M.: A Collection of Mathematical Problems. Interscience Publishers, New York (1968)

29. Vityuk, A.N.: On solutions of hyperbolic differential inclusions with a nonconvex right-hand side. (Russian) Ukran. Mat. Zh. 47(4), 531-534 (1995); translation in Ukrainian Math. J. 47 (1995), no. 4, 617-621 (1996)

30. Vityuk, A.N.; Golushkov, A.V.: Existence of solutions of systems of partial differential equations of fractional order. Nonlinear Oscil. 7, 318-325 (2004) 